University of Nebraska - Lincoln

DigitalCommons@University of Nebraska - Lincoln

Industrial and Management Systems

Industrial and Management Systems

Engineering Faculty Publications

Engineering

1997

\title{
Simulating Queue Scheduling Policies for a Spacecraft Simulator
}

\author{
Paul Savory \\ University of Nebraska at Lincoln, psavory2@gmail.com \\ Gene Saghi
}

Follow this and additional works at: https://digitalcommons.unl.edu/imsefacpub

Part of the Industrial Engineering Commons, Operational Research Commons, Other Operations Research, Systems Engineering and Industrial Engineering Commons, and the Systems Engineering Commons

Savory, Paul and Saghi, Gene, "Simulating Queue Scheduling Policies for a Spacecraft Simulator" (1997). Industrial and Management Systems Engineering Faculty Publications. 49.

https://digitalcommons.unl.edu/imsefacpub/49

This Article is brought to you for free and open access by the Industrial and Management Systems Engineering at DigitalCommons@University of Nebraska - Lincoln. It has been accepted for inclusion in Industrial and Management Systems Engineering Faculty Publications by an authorized administrator of DigitalCommons@University of Nebraska - Lincoln. 


\section{Simulating Queue Scheduling Policies for a Spacecraft Simulator}

P'AUL Savory

Industrial and Manage

University of Nebraska

Lincoln, Nebraska 68588-0518

GENE SAGHI

Advanced Designs Inc.

1495 Garden of the Goils Road

Colorado Springs, Colorato 80907

The mission of the Cassini spacecraft is a unique opportunity to gain insights into major scientific questions about Saturn, the creation of the solar system, and the conditions that led to life on Earth. In preparation for the launch, NASA's Jet Propulsion Laboratory developed a software simulator to emulate the hardware data-control system of the spacecraft. Its primary use is to validate and verify commands that will be transmitted to the spacecraft. To improve the performance of the simulator, we used the SIMAN simulation language to model numerous queue-scheduling policies. Implementation of the simulation results combined with a software-code redesign has greatly improved the performance of the simulator's key control module.

To learn more about Saturn and our 1 solar system, the National Aeronautic and Space Administration (NASA), the European Space Agency, and the Italian Space Agency have formed an international venture to develop the Cassini

(kə-'sē-nē) spacecraft. Cassini is the bestinstrumented planetary probe ever developed, and it is scheduled for launch in

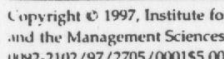

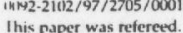

INTERFACES 27: 5 September-October 1997 (pp. 1-8)
October 1997. (The home page http:// www/jpl.nasa.gov/cassini contains a deailed description of the Cassini mission.)

During its mission, ground control personnel will communicate with the spacecraft by sending it sequences of commands. Each sequence instructs the spacecraft to carry out some operation. Examples include firing a thruster to perform a SIMULATION-APPI.ICA TIONS 
maneuver, turning a scientific instrument on or off, and transmitting information back to Earth. With any spacecraft, a sequencing oversight can be costly and tim consuming to resolve. In addition, a sequencing error could cause a catastrophic failure of the spacecraft. To validate and verify that sequences will operate as intended, NASA's Jet Propulsion Laborator has developed a high-speed simulator (HSS) or software spacecraft of the spacecraft's data control system. The HSS will allow ground personnel to test and fix all important command sequences before they upload them to the spacecraft.

The spacecraft's data-control system consists of 31 hardware components.

The spacecraft's data-control system consists of 31 hardware components. The HSS provides bit-level emulators or hardware models for each component. Exam ples include the 1750a central processing unit (CPU), the Reed-Solomon downlink (RSDL) that is responsible for sending telemetry data to the radio transmitter, and the solid-state data recorder (SSR) for storing information.

The scheduler is the key control module in the HSS. It is responsible for executing the 31 hardware models using multithread programming techniques. This programming approach uses threads to execute the hardware models concurrently. A computer process can be thought of as a running computer program and all the state information associated with it. A thrend is an independent stream of control within computer process [Lewis and Berg 1996].
Traditionally, a computer process has a single thread of control and possesses sole ownership of the computer processor's memory and other resources. In a multithreaded environment, a computer process can have many concurrently active threads sharing the process's memory and resources [Kleiman, Shah, and Smaalders 1996]. Using this multithreaded approach, the scheduler must determine the order of model execution and the degree to which models are executed concurrently. task was to improve the performance of the scheduler.

\section{Scheduler Operation}

The hardware components of the datacontrol system on the spacecraft are not independent and must pass messages and information to one another. The spacecr accounts for this dependency by having all data-control components stop and synchronize with one another based on realtime interrupts (RTIs). On the spacecraft, an RTI occurs every eighth of a second. To mimic this operation, the HSS scheduler divides the total run time of a simulation into a series of RTIs. The HSS does not necessarily run in real time. In fact, our results show that it currently takes longer than an eighth of a second to simulate an RTI. As a result, one of the outputs we sought in our analysis was the estimated run time for the HSS to simulate a series of RTIs.

The HSS begins a simulation with each of the 31 models waiting in a queue calle the model queue until an available thread can be assigned to process it for After a thread processes a model for a single RTI, the model is released by the thread and waits in another queue called

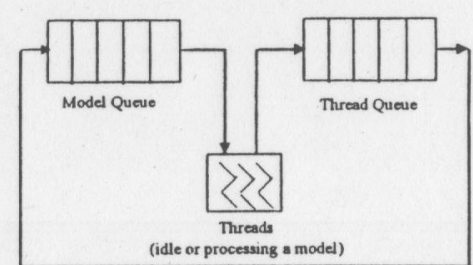

Figure 1: Models wait in the model queue until they are processed by one of the available threads. After processing, models wait in the thread queue until all the remaining models have been processed.

the thread queue until all the other models have been run. A freed thread selects the next model from the model queue and executes it (Figure 1). Thread assignment and model execution continue until all models have been run for one RTI. Once all models have been run, they are released from the thread queue and are sent to the model queue so that they are available to be executed for another RTI. This process continues until the HSS has run a user-specified number of RTIs (Figure 2). Modeling the Scheduler

Our objective was to improve the performance of the scheduler. After a thorough review, we decided to focus on how the scheduler assigns threads to execute the hardware models; that is, what is the best queuing policy for the model queue? One approach for comparing various queuing policies is to implement a new policy in the actual software code of the scheduler, run the HSS, and test for improvement. We judged that such an ap-
Model 1 Model 2 Model 3 Model 4 Model 5

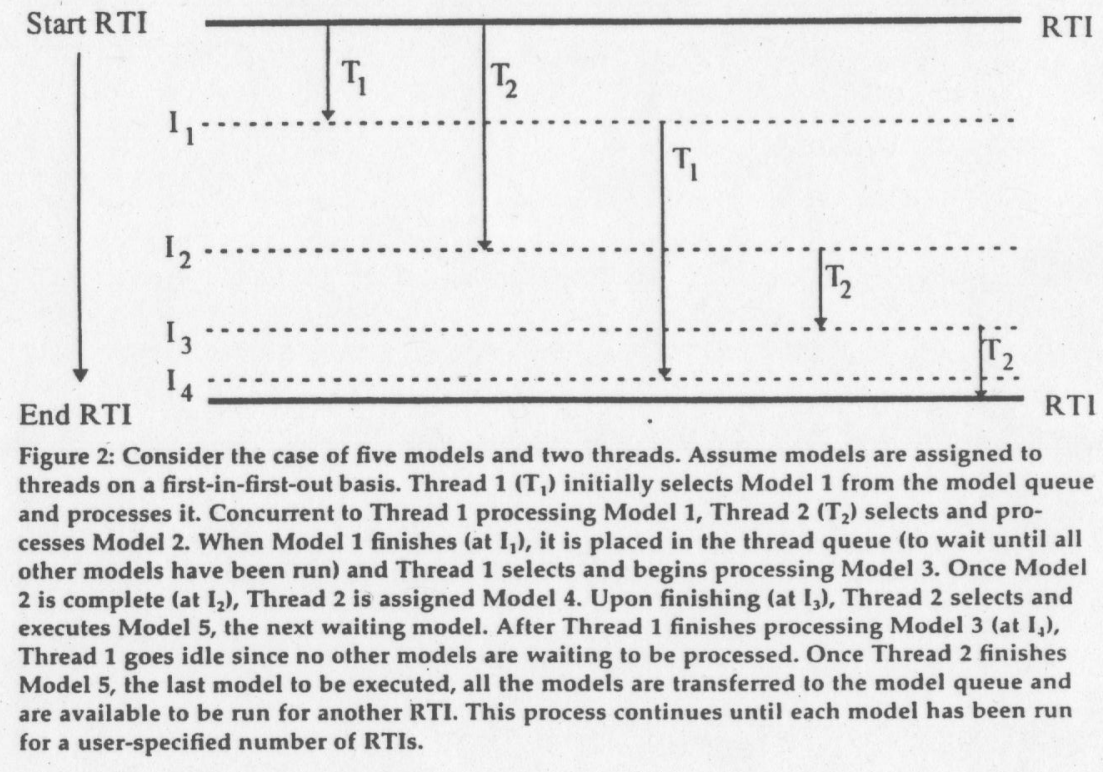


proach was too time intensive and susceptible to coding errors. As an alternative, we chose to create a model of the scheduler. Even though the scheduler is a simple queuing network, developing an analytica queuing model was not feasible. We did

Our best option was to develop a discrete-event simulation model.

not want to limit our analysis to only standard queuing priority rules (for example, first-in-first-out, last-in-first-out) or to make simplifying Markovian assumptions about the processing time distributions describing the hardware models. For maximum flexibility, our best option was to develop a discrete-event simulation model of the scheduler.

Experimental Design

Our first task in building the simulation model was to define our simulation objectives and to specify the factors to be varied. The performance variables to be collected by the simulation model were (1) the total estimated time for the HSS to execute all 31 models, (2) the average number of models waiting in the model queue, and (3) the average number of models waiting in the thread queue. The factors to be varied included the queuing policy for the model queue and the number of threads available to process the hardware models.

We began with an initial list of 14 queuing policies: (1) priority given to the mode that arrives first to the model queue; (2) priority given to the model that arrives last to the model queue; (3) priority given to the model that has been in process the

shortest time; (4) priority given to the model that has been in process the longest time; (5) priority given to the model that

has waited the least total time at the model queue for a thread; (6) priority given to the model that has waited the most total time at the model queue for a thread; (7) priority given to the model that has waited the least total time in the thread queue for all the other models to be completed; (8) priority given to the model that has waited the most total time in the thread queue for all the other models to be completed; (9) priority given to the model that has waited the least time in both the model and thread queues; (10) priority given to the model that has waited the most time in both the model and thread queue; (11) priority given to the model that has the smallest sum of model-queuewaiting time and thread-processing time; (12) priority given to the model that has the largest sum of model-queue-waiting time and thread-processing time; (13) priority given to the model that has the smallest sum of thread-queue-waiting time and thread-processing time; and (14) priority given to the model that has the largest sum of model-queue-waiting time and thread-processing time.

The number of available threads for processing the hardware models is a parameter entered by a user of the HSS. It value is influenced by the processing speed and current utilization of the computer. We wanted to analyze the scheduler's performance as a function of the queuing policy with one, two, three, four, eight, 12, or 16 threads. An obvious question is, since there are 31 models, why not declare 31 threads, one to run each model?
The key disadvantage of this approach is that the majority of those threads will have a low utilization since most of the hardware models have short processing times. While it is true that some marginal improvement is achieved by adding more threads, it is negated by the overhead involved in managing the threads.

Building the Model

The key task in modeling the scheduler is estimating the time for the HSS to execute each of the 31 models for one RTI.

Unfortunately, because the execution time for many of the models is low, we felt that collecting timing estimates for randomly chosen single RTI intervals could be biased by both rounding errors and the actual time collection routines. We decided instead to sample the execution time needed to run each model for an interval of 10 RTIs. We adjust for the timing difference in our simulation model.

To create a sample population of data, we ran the HSS for 1,000 RTIs. We chose at random 35 nonoverlapping intervals of 10 RTIs from within this range. For each of the chosen periods, we collected data on the length of time for the HSS to run 1 Thread 2 Threads 3 Threads

$\begin{array}{llllllll}\text { Policy } 1 & 76.4 & 42.1 & 38.2 & 23.5 & 23.0 & 22.9 & 22.9\end{array}$

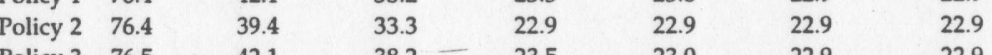

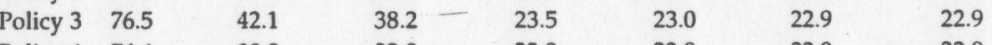

$\begin{array}{llll}\text { Policy } 4 & 76.4 & 38.8 & 32.8\end{array}$

$\begin{array}{llll}\text { Policy } 5 & 76.4 & 41.3 & 37.9\end{array}$

$\begin{array}{llll}\text { Policy } 6 & 76.4 & 41.3 & 35.1\end{array}$

$\begin{array}{llll}\text { Policy } 7 & 76.4 & 40.4 & 33.2\end{array}$

Policy $8 \quad 76.4 \quad 42.1$

$\begin{array}{lll}42.1 & 38.2 & 22.9\end{array}$ Table 1: Average execution time (in seconds) for the HSS to execute each of the 31 hardware with one thread and Policy 1 (first-in-first-out), the HSS will have an estimated execution time of 76.4 seconds. That is, it will take the HSS 76.4 seconds to simulate 12.5 seconds (100 RTI $\times$ 1/8 second per RTI) of the spacecraft data control system. 


\section{$\because \forall$ SAVORY, SAGHI}

the need for validating a simulation model. The Simulation Results

We next ran 40 replications of each of the remaining 56 simulation models (eight remaining queuing policies $\times$ seven thread combinations). We ran each replication until all of the 31 hardware models were simulated for 100 RTIs (Table 1)

\section{We created 98 specific} simulation models.

Using the simulation results, we performed hypothesis tests at each thread value to test if a statistical difference exist among the mean run times for the different queuing policies. For the case of one thread, an analysis of variance showed no statistical differences in the mean execution times of the eight queuing policies. For two, three, and four threads, statistical differences do exist among the queuing policies. Further analysis revealed that for these thread values, the best performing policy is Policy 4 (priority to model that has been in process the longest), followed by Policy 7 (priority to model that has waited the least for other models to finish), and the Policy 5 (priority to model that has waited the least amount of time at both queues).

For eight, 12, and 16 threads, there is no statistical difference in the execution time for the different queuing policies. Although we did not collect data on it, we hypothesize that thread utilization will differ for the queuing policies. That is, the more time-intensive queuing policies (for example, Policy 3) will have a higher use of threads than the less time-intensive policies (for example, Policy 4). Overall, after four threads, adding more threads has a minimal impact on the run time, but will affect the utilization of the threads. As discussed previously, adding more threads can potentially increase the HSS execution time since the computer will have to spend more time managing the threads. Our simulation model did not model the time the computer spends managing threads.

The other two performance variables for the simulation model were the average number in each of the queues. The results for the top three performing queuing policies when four threads are implemented show that the total number of models waiting in both queues are fairly close (Figure 3). The queuing policy affects only which of the two queues is larger. As would be expected, with more than four threads, the average number waiting in both queues dramatically decreases. Conclusion

Our work to improve the performance of the scheduler in the high-speed simulator provided us with the opportunity to

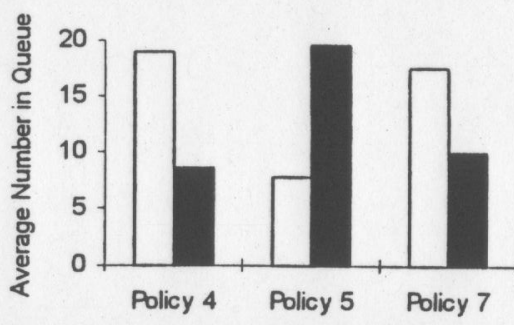

Figure 3: The average number in the model queue (white bar) and the thread queue (black bar) for the top three queuing policies
(whene when four threads are available. Even though policies 4 and 7 are similar, the total number waiting in simulate the simulator. The best performing policy under varying thread values is Policy 4 (schedule the longest running model first). As a result of our analysis, the scheduler module was rewritten to implement Policy 4 as the queuing policy for the model queue. This, combined with a redesign of the software code, has increased the scheduler's performance by 33 percent.

This increased the scheduler's performance by 33 percent.

Unfortunately, there was not a corresponding reduction in the HSS run time. This is primarily the fault of the two hardware models of the central processing unit (CDC_efc_A and CDC_efc_B). These models are bit-level software emulators of the actual $1750 \mathrm{CPU}$ chips, and their execution times dominate the overall run time needed by the HSS. The Jet Propulsion Laboratory is currently working to improve the performance of these timeintensive hardware models. These potential improvements point out a significant limitation of our model. Our analysis is based on the as-is implementation of the HSS. Improving the hardware model execution times may potentially change the processing-time distributions used to describe them (appendix). After the hardware model changes occur, it would be appropriate to rerun the simulations using new processing-time data to confirm the best queuing policy. However, we expect that these changes will have little overall impact on the queuing policy. For this reason, and because of time and budget constraints, it is unlikely that we will rerun our simulation. Acknowledgments

Our participation was supported through summer faculty fellowships provided by the American Society of Engineering Educators (ASEE) in combination with the National Aeronautic and Space Administration (NASA). We thank Kirk Reinholtz and Suzanne Dodd of the Jet Propulsion Laboratory (JPL) for their support of our efforts. We also acknowledge the assistance of Jeffrey Nesheiwat, a doctoral student at Rensselear Polytechnic Institute, who worked on our project as part of his summer co-op position with JPL. APPENDIX

APPENDIX Hacessing Time Distre Models

The probability distributions (in sec-

onds) developed for each of the hardware models are: (1) CDS_efc_A

NORM(1.5,0.1093); (2) CDS_efc_B

$\operatorname{NORM}(1.83,0.1344)$; (3) BC_A:

$\operatorname{EXPO}(0.02203)$; (4) BC_B: $\operatorname{EXPO}(0.01962)$;

(5) RSDI A: LOGN(0.002363,0.0002643);

(5) RSDLA: LOGN

(6) RSDL_B: LOGN $(0.001747,0.00019$

(7) FDU_A: ERLA(1.79e-005,25); (8)

FDU_B: ERLA $(8.3+\mathrm{e}-006,25)$; (9) CRC_A

CRC_B:

$(0.04+\operatorname{LOGN}(0.008891,0.0004655)) ;(11)$

SPAREO: WEIB $(0.0006766,33.1)$; (12)

XBA_A: WEIB $(0.0005171,25.3)$; (13)

XBA_B: WEIB $(0.0004958,17.2)$; (14) PPS_A:

WEIB $(0.0005386,7.1)$; (15) PPS_B:

ERLA(1.67e-005,25); (16) RFS

WEIB $(0.0004882,148)$ (17) CAPS:

ERLA(1.3e-005,25); (18) AACS_A_XBA:

ERLA(1.3e-005,25); (18) AACS_A_X
$\left(1.25^{*}\right.$ NORM(1.5,0.1093)); and (19)

(1.25*NORM(1.5,0.1093)); and (19)

Time values were not available for models

(18) and (19) but HSS developers esti-

mated them as a function (1.25 times) of

the CDS_efc_A (model 1) and CDS_efc_B 
(model 2) distributions. The probability distributions for the remaining hardware models are: (20) CDA: ERLA(1.27e-005,25); (21) PMS_B: ERLA(1.31e-005,25); (22) EU_A: ERLA(1.63e-005,25); (23) EU_B: ERLA(1.75e-005,25); (24) RSP_A: ERLA(21.8e-005,25); (25) RSP_B: ERLA(1.62e-005,25); (26) PSA_A: ERLA(1.49e-005,25); (27) PSA_B: ERLA(1.32e-005,25); (28) ISS_NAC: ERLA(1.26e-005,25); (29) ISS_WAC: ERLA(1.37e-005,25); (30) SSRIU_A: LOGN(0.003906,8.93e-005); and (31) SSRIU_B: WEIB $(0.002747,58.2)$ References

Kleiman, Steve; Shah, Devang, and Smaalders, Bart: 1996, Programming with Threads, SunSoft Press, Mountain View, California.

Lewis, Bill and Berg, Daniel J. 1996, Threads Primer: A Guide to Multithreaded Programming, SunSoft Press, Mountain View, California. Pegden, C. Dennis; Shannon, Robert E., and Sadowski, Randall P. 1995, Introduction to Simulation Using SIMAN, McGraw-Hill, New

Michael H. Jahan, Technical Supervisor (Acting), Ground System Engineering Group, Jet Propulsion Laboratory, California Institute of Technology, 4800 Oak

Grove Drive, Pasadena, California 91109 8099 , writes, "As part of the NASA

Ground Systems Engineering group at the Jet Propulsion Laboratory, Dr. Paul Savory worked with another ASEE/NASA summer faculty fellow (Eugene Saghi) and a doctoral co-op student (Jeffrey Nesheiwat) to optimize the performance of the scheduler (one of the main control programs) in the Cassini high-speed simulator. In addition to a redesign of the scheduler code they used a discrete-event simulation model to model the scheduler's operation. They specifically explored testing various queue priority rules for how threads are assigned to models needing to be exe- ched. The key objective was to improve the performance of the scheduler.

"As a result of their efforts, the scheduler module has been rewritten and integrated into the latest version of the highspeed simulator." 